\title{
Sommer trifft Schulpraxis
}

\section{Inklusive Einblicke aus der Retrospektive}

\section{DOI: https://doi.org/10.53349/sv.2021.i1.a75}

Was passiert, wenn der Sommer auf die Schule trifft? Ein Erfolgsprojekt, das Bildungsnachteilen entgegenwirkt und das seinesgleichen sucht, entsteht in Zeiten einer weltweiten Pandemie. Die Sommerschule 2020 ist gekommen, um zu bleiben. Der sommerliche Expansionskurs bedeutet gleichermaßen die Erweiterung der Schularten wie die Erweiterung der Fächer. Was passiert zudem, wenn die Sommerschule auf die Schulpraxis trifft? Eine kongeniale Freundschaft entsteht, welche die Pädagogischpraktischen Studien sommerlich qualitätsvoll ergänzt. Über diese besondere Freundschaft, mit dem Fokus auf chancengerechte Bildung, seien im Zuge des Beitrages inklusive Einblicke aus Studierendensicht geboten.

Bildung, Sommer, Schule, Schulpraxis

„Einer ist groß, einer ist klein, und du darfst rein ..."1
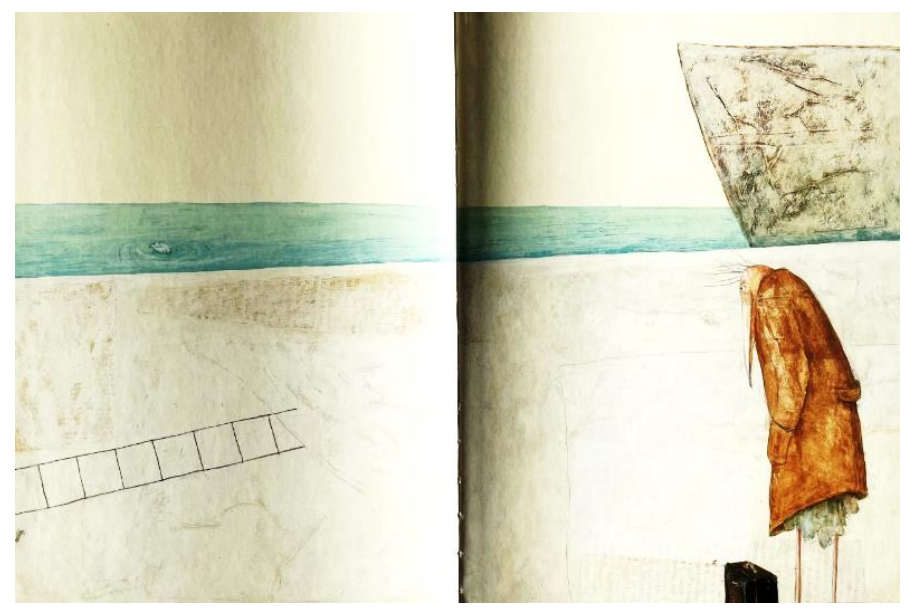

Abbildung 1: Janisch \& Bansch 2010 | @ Jungbrunnen 
Als eindeutiges Erfolgsprojekt expandiert die Sommerschule mit Sommer 2021. Bereits 35.000 Schüler*innen sind heuer für die Sommerschule angemeldet. Insgesamt haben sich 760 Schulstandorte als Sommerschulstandorte für die Durchführung der Sommerschule gemeldet. Deutsch als Unterrichtssprache steht noch immer im Fokus des Sommerschulgeschehens, jedoch wird in der Sommerschule 2021 auch Mathematik projektorientiert unterrichtet sowie in der Volksschule auch Sachunterricht. Freiwillig und kostenlos stellt sich die Sommerschule abermals vor und fließt positiv in die Mitarbeitsnote ein.

Die Sommerschule findet in den letzten beiden Ferienwochen statt: In den östlichen Bundesländern von 23. August bis 3. September, im Westen von 30. August bis 10. September. In der Oberstufe finden die Kurse auch nur eine Woche lang statt.

Der Unterricht in der Sommerschule hebt sich in der Primarstufe und in der Sekundarstufe I vom ganzjährigen Unterricht ab. Am Beginn steht ein Tag des Kennenlernens, da die Gruppen zumeist heterogen und oft standortübergreifend sind. Der Unterricht verläuft in einem Abwechslungsspiel themenzentriert, lehrerzentriert und projektorientiert. Alternierend gibt es fachlichen und überfachlichen Unterricht. Im Zentrum der Sommerschule steht die Stärkung des Sprachbewusstseins in der Unterrichtssprache Deutsch im Bereich des Lesens, Schreibens, Hörverstehens und Sprechens sowie der Erwerb von mathematischen Grundkompetenzen und sachunterrichtliche Vertiefung. Alle Schüler*innen nehmen an allen Schulfächern im Sinne einer Projektorientierung teil.

Hauptsächlich gestalten Studierende der Universitäten und Pädagogischen Hochschulen den Sommerschulunterricht. Sie bekommen für ihre Tätigkeit an der Sommerschule 5 ECTS-Anrechnungspunkte sowie eine Materialentschädigung. Zusätzlich unterrichten auch Lehrkräfte auf freiwilliger Basis, und Buddies unterstützen an den Sommerschulen. Diese Schüler*innen der Oberstufen können sich freiwillig für diese Tätigkeit melden. Der Einsatz der Schulleitungen und der pädagogischen Teams vor Ort macht die Sommerschule erst möglich, neben dem engagierten Einsatz der Studierenden!

\section{„Eine bleibt stehen, einer geht vorbei, schon sind wir zwei."}

Seitens der Pädagogischen Hochschule Niederösterreich nehmen an der diesjährigen Sommerschule 2021 über 150 Studierende teil, darunter die beiden Studierenden Sonja Reisinger und Daniel Osztovics. Bereits im Rahmen der Sommerschule 2020 waren die genannten Studierenden unterrichtend an Sommerschulstandorten tätig. Im Zuge der Pressekonferenz des BMBWF, am 17. Juni 2021, gaben sie inklusive Einblicke in die wahrgenommene Erfahrung der Sommerschule als Schulpraxis mit Mehrwert, die einen wichtige Ergänzung zu den Pädagogisch-praktischen Studien darstellt.

Die Sommerschule 2020 hat Daniel Osztovics an den Sommerschulstandort der Volksschule Berndorf geführt. Die Volksschule Kottingbrunn wird sein diesjähriger Sommerschulpraxis- 


\section{\# schuleverantworten}

standort sein. Nachfolgend führen leitende Fragen, die auf den Artikel „Sommer macht Schule - Bildung für alle!?“ dieser Ausgabe verweisen, durch die Wahrnehmungen der beiden Studierenden.

\section{„Eine sagt Huhn, einer sagt Ei, jetzt sind wir drei.“}

Ab drei Kindern und Jugendlichen ist grundsätzlich von einer Gruppe zu sprechen. Im Kontext der Sommerschule wird auf Gruppengrößen von acht bis zwölf Lernenden geachtet. Dabei steht Individualisierung und Differenzierung auf der sommerschulischen Tagesordnung, da die Zielgruppe ein höchstmögliches $M a ß$ an Heterogenität aufweist. Bundesminister Heinz Faßmann verwies im Zuge der Pressekonferenz u.a. auch auf die Teilnahme von 600 Schüler*innen mit sonderpädagogischem Förderbedarf.

Wie wird Individualisierung und Differenzierung wahrgenommen?

Wir Studierende übernehmen für zwei Wochen - alleine oder zu zweit im Tandem-die komplette Verantwortung für eine ganze Gruppe - zugegeben eine sehr heterogene Gruppe, zumindest war es bei mir letztes Jahr so. Es sind nicht nur unterschiedliche Schulstufen dabei, sondern auch in derselben Altersstufe unterschiedliche Leistungsniveaus: einerseits Kinder, die noch Probleme bei der Wort-Bild-Zuordnung haben, andererseits Kinder, die ganze Sätze fehlerfrei sprechen und schreiben können. Du hast in der Sommerschule wirklich die Möglichkeit, die Kinder individuell zu fördern und vor allem auch gezielt zu fördern.

\section{„Einer spielt Flöte, einer Klavier, da sind wir vier.“}

Im Rahmen der Sommerschule im Bereich der Primarstufe können die Lerngruppen heterogen aus allen vier Schulstufen zusammengesetzt sein.

\section{„Einer läuft barfuß, die andre braucht Strümpf, gleich sind wir fünf.“}

Wird die Vorschulstufe mitgerechnet, so ergibt sich die Vielfalt von eigentlich fünf Schulstufen, die im Rahmen der Sommerschule miteinander lernen.

\section{"Eine liebt Eis, eine nur Keks, und wir sind sechs."}

Der projektorientierte Unterricht, der mit Lernen konnotiert sein soll, sich jedoch vom ganzjährigen Fächerkanon abheben darf, kann aus mehreren kleinen Projekten bestehen, die unter einem übergeordneten Thema zusammengeführt werden.

Welche Praxiserfahrung stellt der Ergänzungsunterricht im Sinne eines projektorientierten Unterrichts dar?

Man kann viel Praxiserfahrung sammeln, kreativ sein, ein Projekt planen, Verantwortung übernehmen und eine Gruppe zwei Wochen lang intensiv begleiten. Das Ganze ist projektori- 


\section{\# schuleverantworten}

führungskultur_innovation_autonomie

entiert und soll den Schülerinnen und Schülern einen guten Start in das neue Schuljahr ermöglichen. Es hat mir sehr viel Spaß gemacht, die Kinder auf das nächste Schuljahr vorzubereiten. Anhand einer Projektmappe, die sich aus den Arbeitsblättern und erarbeiteten Materialien ergab, die sich im Laufe der zwei Wochen ansammelten, versuchten wir die Nachhaltigkeit der Sommerschule zu sichern.

\section{"Einer ging fort, ein anderer ist geblieben, nun sind wir sieben."}

Natürlich wären sieben Wochen beispielsweise besser als nur zwei, um den Aufholbedarf zu kompensieren, der sich durch die Schulschließungen hinsichtlich des Lernstoffes ergeben hat. Jedoch sind zwei Wochen ein guter Anfang, um auf den Schulstart vorzubereiten.

Wie wird die Lernzeit im Kontext der Dauer der Sommerschule bewertet?

Natürlich kann man in zwei Wochen nicht ein ganzes Schuljahr nachholen, aber man kann wirklich viel weiterbringen in den zwei Wochen. Und ich war erstaunt, wie viel die Kinder am Ende der Sommerschule auch mitgenommen haben. Du hast in der Sommerschule wirklich die Möglichkeit, die Kinder individuell zu fördern und vor allem auch gezielt zu fördern. Die Kinder konnten in diesen zwei Wochen viel lernen und man sah bei der Präsentation am letzten Sommerschultag eine deutliche Verbesserung.

\section{„Einer freut sich auf den Tag, die andre auf die Nacht, schau, wir sind acht.“}

Wer hätte das gedacht? Auch Studierende engagieren sich in der unterrichtsfreien Zeit, genannt Ferien, im Sinne eines gesamtgesellschaftlichen Auftrages für Schüler*innen, die von Bildungsnachteilen betroffen sind, und leisten damit einen wichtigen Beitrag, eben diesen entgegenzuwirken.

Wie wird Bildungsgerechtigkeit im Sinne von chancengerechter Qualifikation im Kontext der Sommerschule wahrgenommen?

Es ist nicht nur eine Chance für uns Lehramtsstudent*innen, sondern vor allem für die Schüle$r^{*}$ innen. Denn es darf einfach nicht sein, dass es wegen der Pandemie Nachteile für die Schüler*innen gibt.

Wie wird die Wichtigkeit der Förderung der Unterrichtssprache Deutsch empfunden?

Die Kinder haben unter Corona sehr gelitten und jetzt heißt es Ärmel hochkrempeln und allen, die Probleme mit der Unterrichtssprache haben, oder die drohen, den Anschluss in der Klasse womöglich zu verlieren, wieder ins Boot zu holen, den Folgen der Schulschließungen entgegenzuwirken und die Schüler*innen bestmöglich auf den Unterricht im nächsten Schuljahr vorzubereiten.

Wir hatten bereits letztes Jahr - neben dem Schwerpunkt Deutschförderung - auch Mathematik und Sachunterricht dabei - diese Gegenstände werden heuer noch mehr einfließen. 


\section{\# schuleverantworten}

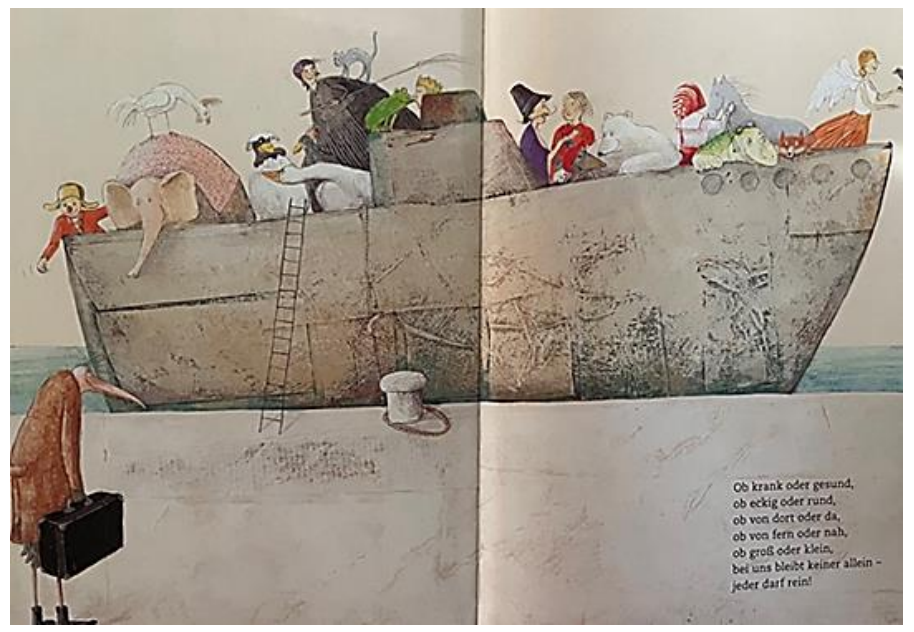

„Ob krank oder gesund, ob eckig oder rund, ob von dort oder da, ob von fern oder nach, ob groß oder klein, bei uns bleibt keiner allein jeder darf rein!“

(Janisch \& Bansch 2010)

Abbildung 2: Janisch \& Bansch 2010 | @ Jungbrunnen

Nicht nur für die Schüler*innen der Sommerschule stellt die Sommerschule einen Mehrwert dar, sondern auch für die Studierenden, die Resümee ziehen:

Man kann viel Praxiserfahrung sammeln, kreativ sein, ein Projekt planen, Verantwortung übernehmen und eine Gruppe zwei Wochen lang intensiv begleiten.

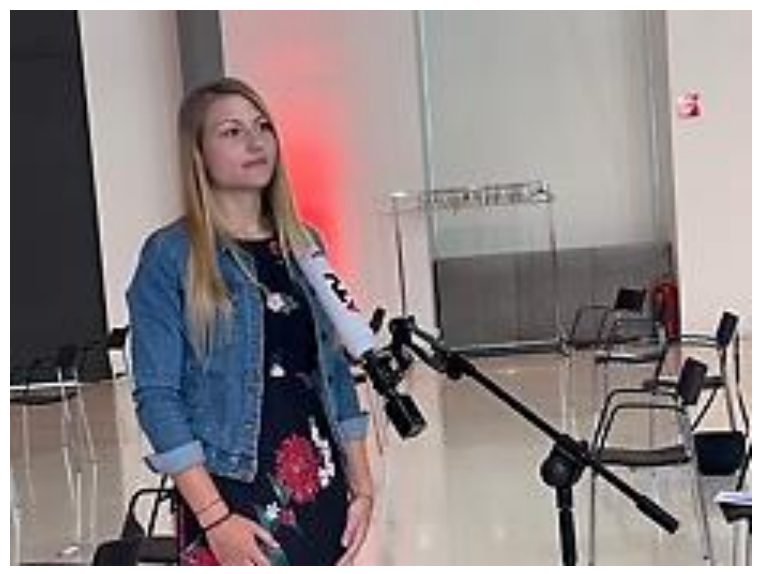

Abbildung 3: PH NÖ-Studierende Sonja Reisinger: „Ich freue mich auch dieses Jahr wieder sehr auf die Sommerschule und die Schüler*innen, welche ich gemeinsam mit einer Studienkollegin unterrichten werde." | Foto: PH NÖ/Zechner

\section{„Eine ist traurig, einer kann sich freun, das macht schon neun."}

Auch der Sommer 2021 wird vorüberziehen und somit die kommenden neun Wochen Sommerferienzeit. Doch Dank der Bildungsinitiative von Bundesminister Heinz Faßmann können sich alle Protagonist*innen der Sommerschule freuen: „Die Sommerschule ist gekommen, um 


\section{\# schuleverantworten}

führungskultur_innovation_autonomie

zu bleiben", so BM Faßmann im Zuge der Pressekonferenz, und dies für eine jährlich erweiterte Zielgruppe, in Annäherung an den Buchtitel, der durch diese Einblicke geführt hat: Und du darfst rein! (Janisch \& Bansch 2010)

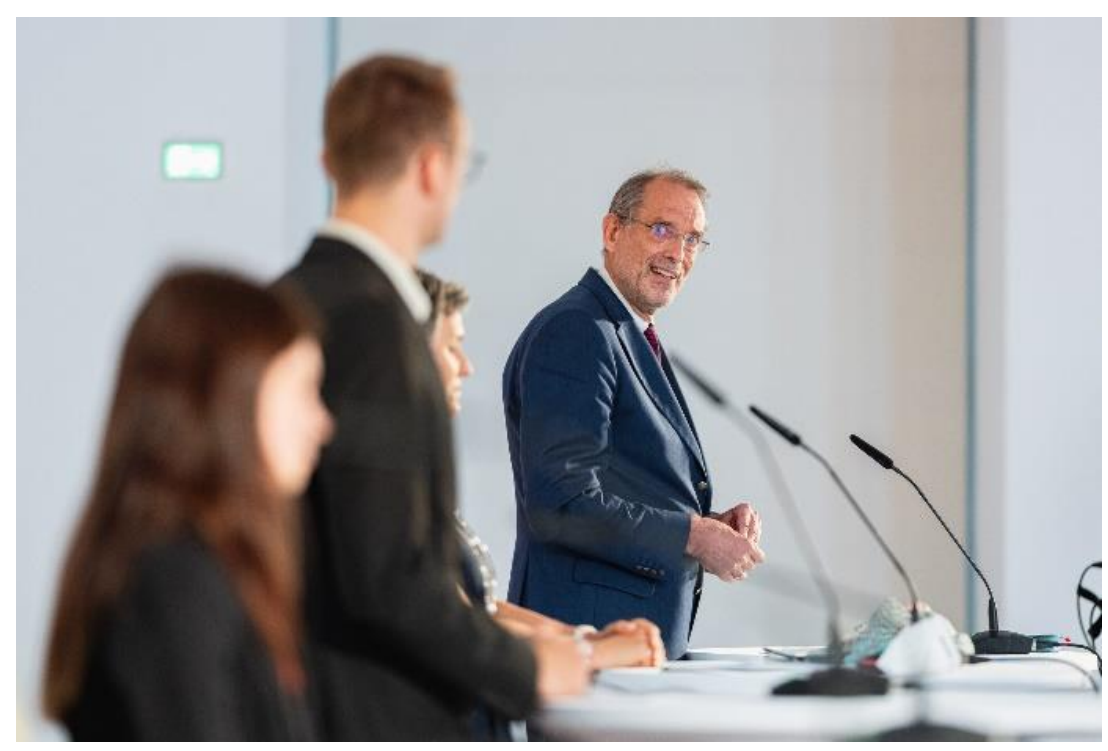

Abbildung 4: Im Rahmen einer Pressekonferenz präsentierten Bildungsminister Heinz Faßmann, Uni Wien-Vizerektorin Christa Schnabel, PH-NÖ-Studierender Daniel Osztovics, Buddy Lea-Marie Pautsch und Schülerin Lena Kolarik die Sommerschule 2021. | Foto: BKA

\section{Literaturverzeichnis}

BMBWF 2021: Pressekonferenz zur Sommerschule. https://www.facebook.com/wissensministerium/videos/517431419449011 |, Stand vom 17.06.2021

Fikisz, W. (2021). Pressetext zur Pressekonferenz der Sommerschule 2021. https://www.ph-noe.ac.at/no_cache/de/news/news-detail/artikel/sommerschule-studierende-der-ph-noe.html I, Stand vom 19.06.2021

Janisch, H. \& Bansch, H. (2010). Und du darfst rein. Wien: Jungbrunnen.

Osztovics, D. \& Reisinger, S. (2021): Pressekonferenz zur Sommerschule, 17.06.2021.

Rauscher, E. \& Zechner, K. A. (2021, im Druck). Sommer und Schule? Sommerschule als Katalysator zur Verflechtung einer scheinbaren Paradoxie. In C. Kaluza, G. Kulhanek-Wehlend, G. Lauss, J. Majcen, R. Petz, B. Schimek, A. Schnider, S. Severin \& E. Süss-Stepancik (Hg.) (2021). Sommerschule 2020: SUMMERSPLASH - zur wissenschaftlichen Verortung der Sommerschule. Wien: LIT. (Forschungsperspektiven, Sonderband 3).

\section{Anmerkungen}

${ }^{1}$ Alle Zitate der Zwischenüberschriften aus: Janisch \& Bansch 2010. 


\section{Autorin}

Kerstin Angelika Zechner, Mag. phil., MA Bakk. phil.

Seit 2017 an der Pädagogischen Hochschule Niederösterreich; Departmentleiterin für Diversität; davor zwei Jahrzehnte im Bereich der Diversität und schulischen Inklusion tätig, u.a. an der KPH Graz.

Kontakt: kerstin.zechner@ph-noe.ac.at 